\title{
Charge Injection in Gold Ground Electrode Corona Charged Polyethylene Film: Numerical Simulation on Surface Potential Decay
}

\author{
Y. Zhuang, G. Chen and M. Rotaru \\ School of Electronics and Computer Science, University of Southampton \\ Southampton, SO17 1BJ, United Kingdom \\ yz205@ecs.soton.ac.uk
}

\begin{abstract}
Surface potential decay measurement is a simple and low cost tool to examine electrical properties of insulation materials. Bipolar charge injection has been verified by the measurement of space charge in the corona-charged sample using the pulsed electroacoustic method (PEA). Over the years, various models have been proposed to describe charge transport within the material and one common feature in these models is that they were all based on single charge injection from the charged surface. A new model based on a bipolar charge injection has been proposed recently. In the present paper, numerical simulations have been carried out using the model and results have been compared with surface potential decays obtained from $50 \mu \mathrm{m}$ low density polyethylene films and gold coating sample after corona charged and PEA measurements. The simulation results show that several features experimentally observed can be readily revealed.
\end{abstract}

\section{INTRODUCTION}

There has been significant interest shown in surface potential decay of corona charged polymeric materials recently. Various models were proposed to explain the cross-over phenomenon after it had been found in 1967 [1]. It is known that the key factor that influences the surface potential decay process is charge injection. However, most models were established based on single charge injection at high field and applied surface conduction for lower field. It has been found [2] that bipolar charge injection has taken place in corona charged LDPE films by comparing the results with gold ground electrode LDPE films. The results from the pulsed electro-acoustic method (PEA) also proved that bipolar charge injection affect both lower and higher field.

\section{MODEL DESCRIPTION}

Recently, the bipolar charge transport model has widely used to describe the current-voltage characteristics of polymeric insulation. The model contains three important components: charge build-up (or generation), charge transport process with trapping/de-trapping and charge recombination under dc voltage. One of the first attempts to develop a bipolar charge transport model in relation to experimentally determined space charge profiles and their dynamics was proposed by Alison and Hill in 1994 [3].

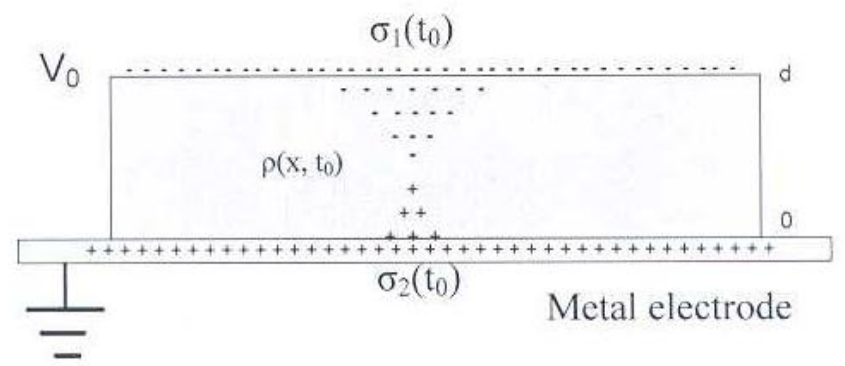

Figure 1. Schematic diagram of surface charge and space charge distribution immediately after corona charging [4].

In Figure $1, V_{0}$ is the initial decay voltage and represents the upper limit of the surface potential. $\sigma_{1}\left(t_{0}\right), \rho\left(x, t_{0}\right)$ and $\sigma_{2}\left(t_{0}\right)$ are surface charge on the top surface, space charge in the sample and induced charge on the metal electrode respectively. The initial values will depend on the charging voltage and time and they are all changed with time.

The boundary condition is defined by the Schottky injection at both electrodes,

$$
j_{e / h}(0, t)=A T^{2} \exp \left(-\frac{e w_{e / h i}}{k T}\right) \exp \left(\frac{e}{k T} \sqrt{\frac{e E(0 / d, t)}{4 \pi \varepsilon}}\right)
$$

Where $j_{e}(0, t)$ and $j_{h}(d, t)$ are the fluxes of electrons and holes at the cathode and anode respectively; $\mathrm{T}$ is the temperature; $A=1.2 \times 10^{6} \mathrm{Am}^{-1} \mathrm{~K}^{-2}$ is the Richardson constant; $w_{e i}$ and $w_{h i}$ are the injection barrier for the electrons and holes.

The charge transport in the bulk of the sample is determined by the electric field. The model assumes that the field components are represented by $E_{\rho}(t), E_{\sigma l}(t)$ and $E_{\sigma 2}(t)$ respectively. The surface potential across the sample can be calculated by integrating the total electric field:

$$
\mathrm{V}_{0}(\mathrm{t})=\int_{0}^{\mathrm{d}}\left[\mathrm{E}_{\rho}(\mathrm{t})+\mathrm{E}_{\sigma_{1}}(\mathrm{t})+\mathrm{E}_{\sigma_{2}}(\mathrm{t})\right] \mathrm{dx}
$$

In addition, the total charge in the system at any time must in balance, i.e.

$$
\sigma_{1}(\mathrm{t}) \mathrm{S}+\sigma_{2}(\mathrm{t}) \mathrm{S}+\int_{0}^{\mathrm{d}} \rho(\mathrm{x}, \mathrm{t}) \mathrm{Sdx}=0
$$

where $S$ is the surface area where charges are present. 
It is clear to see that $\sigma_{l}(t), \sigma_{2}(t)$ and $\rho(x, t)$ are dependent quantities. Based on the model, it is possible to calculate $\rho(x, t)$ during the corona charging until a predefined charging time $t=t_{0}$. The quantities $V_{0}\left(t_{0}\right)$ and $\rho\left(x, t_{0}\right)$ are the initial condition for surface potential decay. This allows to determine $\sigma_{l}\left(t_{0}\right)$ and $\sigma_{2}\left(t_{0}\right)$ through equations (2) and (3). Once these initial four quantities are determined, one can calculate new space charge distribution $\rho\left(x, t_{0}+\Delta t\right)$ based on the proposed model and the two surface density $\sigma_{l}\left(t_{0}+\Delta t\right)$ and $\sigma_{2}\left(t_{0}+\Delta t\right)$ using the Schottky injection.

\section{SimUlATION RESUlTS AND DISCUSSION}

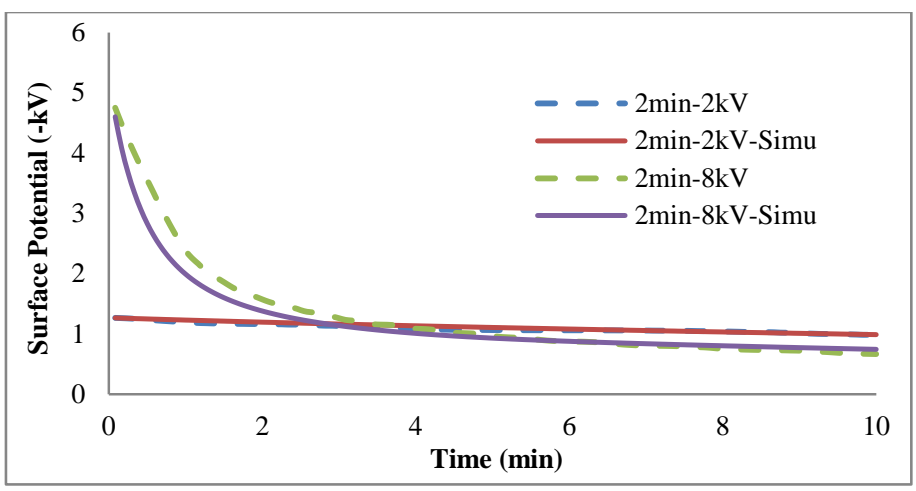

Figure 2. Simulation results compared with experimental results for normal aluminum ground sample.

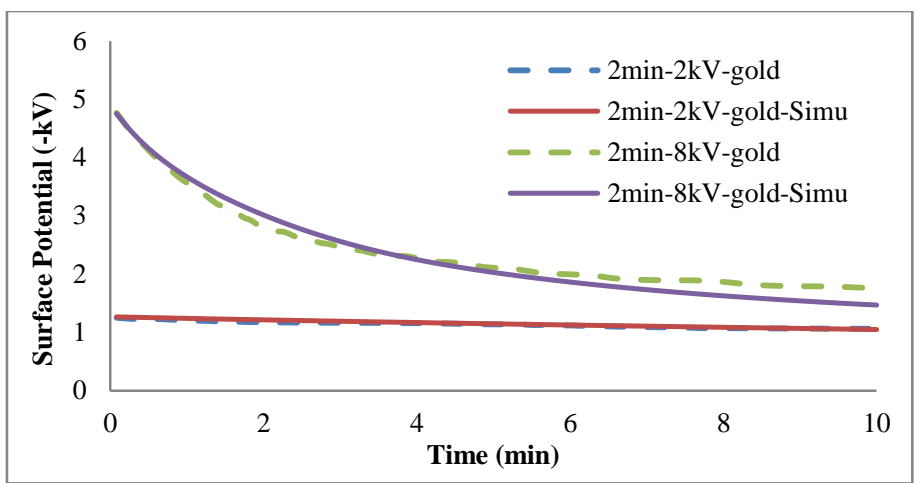

Figure 3. Simulation results compared with experimental results for gold electrode ground sample.

The simulation results compared with experimental results for both lower field and higher field for normal aluminum ground are shown in Figure 2. The results for gold electrode ground are shown in Figure 3. In orger to fit the experimental data, the mobility and barrier height for Schottky injection had to be modified. The values used in our model are shown in Table I. All other parameters in Table II associated with properties of the sample and the environments were kept the same. The mobility during each simulation was kept constant and the injection was controlled by equation (1) as it contains electric field. It can be seen that at lower field, due to the small difference between the initial decay potential and the potential at $10 \mathrm{~min}$, constant mobility can give a perfect match to experimental results; however, at higher field, a small difference can be observed between the experimental and the simulation results at either beginning of the decay process or at the $10 \mathrm{~min}$ when constant mobility was used. This would be explained by the fact that the surface potential drops significantly hence resulting in a big change in electric field. As a result, the simulation results at higher field do not perfectly match the experimental results, but the decay trend is still the same and the cross-over phenomenon can be also observed.

TABLE I

VARIOUS SimUlation PARAMETERS

\begin{tabular}{|c|c|c|c|}
\hline & Mobility $\left(\mathrm{m}^{2} \mathrm{~V}^{-1} \mathrm{~s}^{-1}\right)$ & $w_{e i}(\mathrm{eV})$ & $w_{h i}(\mathrm{eV})$ \\
\hline $2 \mathrm{kV}$ normal & $3 \times 10^{-15}$ & 1.16 & 1.15 \\
\hline $2 \mathrm{kV}$ gold & $3 \times 10^{-15}$ & 1.16 & 1.17 \\
\hline 8kV normal & $1 \times 10^{-14}$ & 1.14 & 1.13 \\
\hline 8kV gold & $3.5 \times 10^{-15}$ & 1.14 & 1.25 \\
\hline
\end{tabular}

TABLE II

Fixed SimUlation PARAMETERS

\begin{tabular}{|c|c|}
\hline Electrons trapping coefficient $\left(\mathrm{s}^{-1}\right)$ & $1 \times 10^{-3}$ \\
\hline Holes trapping coefficient $\left(\mathrm{s}^{-1}\right)$ & $1 \times 10^{-3}$ \\
\hline $\mathrm{S}_{0}\left(\mathrm{~m}^{3} \mathrm{C}^{-1} \mathrm{~s}^{-1}\right)$ & $4 \times 10^{-3}$ \\
\hline $\mathrm{S}_{1}\left(\mathrm{~m}^{3} \mathrm{C}^{-1} \mathrm{~s}^{-1}\right)$ & $4 \times 10^{-3}$ \\
\hline $\mathrm{S}_{2}\left(\mathrm{~m}^{3} \mathrm{C}^{-1} \mathrm{~s}^{-1}\right)$ & $4 \times 10^{-3}$ \\
\hline $\mathrm{S}_{3}\left(\mathrm{~m}^{3} \mathrm{C}^{-1} \mathrm{~s}^{-1}\right)$ & 0 \\
\hline Electrons de-trapping barrier height $(\mathrm{eV})$ & 0.92 \\
\hline Holes de-trapping barrier height $(\mathrm{eV})$ & 0.92 \\
\hline Attempt to escape frequency $\left(\mathrm{s}^{-1}\right)$ & $6 \times 10^{12}$ \\
\hline Relative permittivity of LDPE & 2.3 \\
\hline
\end{tabular}

\section{CONCLUSION}

In conclusion, it can be said that bipolar charge injection in the major mechanism for the surface potential decay and the model successfully simulate the decay process at both lower field and higher field. However, the barrier height for Schottky injection are different at these voltage levels especially for the hole injection from gold electrode ground; the field dependent mobility should be selected to improve current model with a better agreement to experimental results.

\section{REFERENCES}

[1] M. Ieda, G. Sawa and I. Shinohara, A decay process of surface electric charges across polyethylene film, J. Appl. Phys. 6 793-4

[2] Z. Xu, L. Zhang and G. Chen, Decay of electric charge on corona charged polyethylene, J. Phys. D: Appl. Phys. 40 7085-9

[3] J. M. Alison and R. M. Hill, A model for bipolar charge transport trapping and recombination in degassed crosslinked polyethylene, J. Phys. D: Appl. Phys. 27 1291-9

[4] G. Chen, J. Zhao and Y. Zhuang, Numerical modeling of surface potential decay of corona charged polymeric material, 2010 International Conference on Solid Dielectrics, July 4-9, Potsdam, Germany. pp. 549-522 